\title{
Measurements of $\alpha_{s}$ from event shapes and the four-jet rate ${ }^{*}$
}

\author{
G. Dissertori ${ }^{\mathrm{a}}$ \\ anstitute for Particle Physics, ETH Zürich, \\ ETH Hönggerberg HPK E28, CH-8093 Zürich, Switzerland
}

\begin{abstract}
New results from measurements of the strong coupling constant $\alpha_{s}\left(M_{\mathrm{Z}}\right)$ at LEP are presented. In particular, a new LEP combination of results based on event-shape variables has become available, where a new method for the estimation of the theoretical uncertainty has been implemented. Furthermore, two other analyses are quoted, based on power corrections and the four-jet rate.
\end{abstract}

\section{Introduction}

The strong coupling constant $\alpha_{s}$ is the only free parameter of the QCD Lagrangian and many measurement have been performed at various energy scales in different processes in order to determine it precisely [1]. The measurements at LEP have contributed significantly to this effort.

Two years after the closure of LEP still new results from QCD analyses become available [2]. These results are based on data from LEP1, where the centre-of-mass energy was around 91 $\mathrm{GeV}$ and the data sample consisted of several million events, practically background free, as well as from LEP2, with energies from $133 \mathrm{GeV}$ up to $206 \mathrm{GeV}$. Here the data samples are much smaller, of the order of several thousand events, and the backgrounds (mainly fully hadronic Wpair events) can amount up to about $15 \%$. In addition, at LEP2 a special selection has to be applied in order to eliminate the so-called radiative return events, where a hard photon is radiated in the initial state, reducing the effective centre-of-mass energy to the $\mathrm{Z}$ mass. This leads to a significant loss in statistics. Therefore the $\alpha_{s}$ measurements at LEP2 have a much larger statistical uncertainty. However, systematic uncertainties related to non-perturbative corrections or unknown higher order terms are reduced because of the larger energy scale. This motivates the combination of all measurements performed by the four

\footnotetext{
*Talk presented at the 31st International Conference on
} High Energy Physics, Amsterdam, 24-31 July 2002.
LEP experiments ALEPH, DELPHI, OPAL and L3, in order to obtain a precise test of the running of the coupling, as well as a competitive measurement at LEP2 compared to LEP1, and finally the best possible overall combination. Such a combination has now been performed by the LEP QCD working group (LEPQCDWG) for measurements based on event-shape variables.

\section{Event-shape variables}

Event-shape variables are a classical tool to measure the strong coupling constant. They are sensitive to the topology of an event, therefore to gluon radiation and thus to the coupling strength. The most thoroughly tested variables are thrust, heavy jet mass, C-parameter, total and wide jet broadening and the $y_{3}$-distribution, where $y_{3}$ is the resolution parameter where a three-jet event is clustered into a two-jet event (Durham clustering algorithm). For these variables the perturbative QCD predictions exist at next-to-leading order (NLO), $\mathcal{O}\left(\alpha_{s}^{2}\right)$. In addition, large logarithms $L=\ln (1 / y)$ have been resummed at next-toleading logarithmic (NLL) accuracy to all orders in $\alpha_{s}$, where $y$ is an event-shape variable. Only recently some missing NLL terms have been calculated for the $y_{3}$-distribution [3]. Several matching schemes have been proposed over the years in order to combine the NLO and NLL calculations, such as the $\log \mathrm{R}$ and $\mathrm{R}$ matching schemes [4]. The LEPQCDWG adopts so-called modified matching schemes, where the logarithmic term is replaced 
by $L \rightarrow \tilde{L}=\frac{1}{p} \ln \left[(1 / y)^{p}-\left(1 / y_{\max }\right)^{p}+1\right]$. This ensures a physical behaviour of the prediction at the phase space boundary $y_{\max }$. The choice of the power $p$ is arbitrary, in the sense that different choices only give different subleading contributions, whereas the NLL terms remain unchanged. The modified $\log \mathrm{R}$ matching scheme corresponds to $p=1$, whereas $p=2$ is called the second degree modification. As mentioned

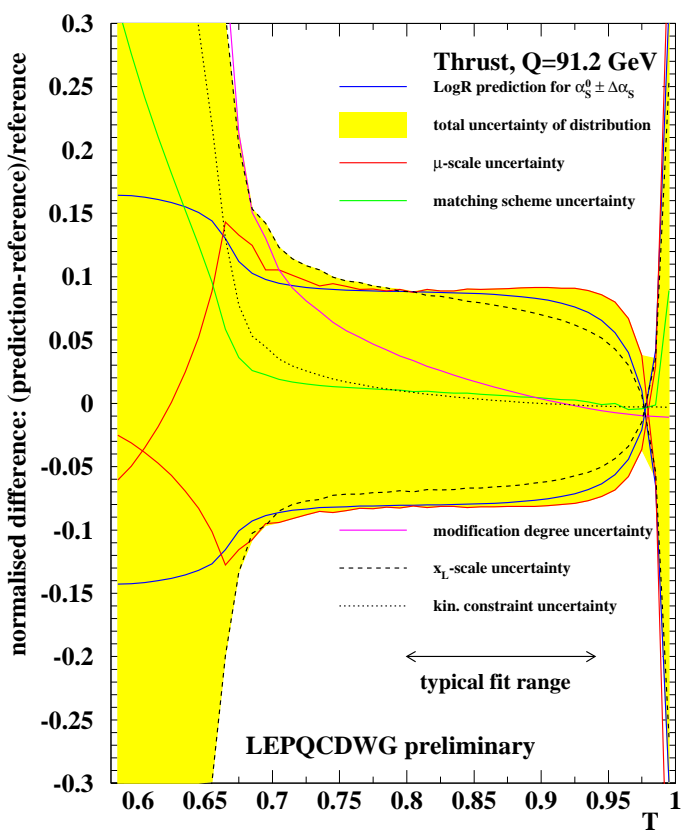

Figure 1. Uncertainty band for the thrust distribution.

earlier, the measurements at LEP1 are basically background free, whereas at LEP2 the W background is very difficult to suppress, in particular in the multi-jet region of the event-shape distribution which is also sensitive to hard gluon radiation. In order to avoid large systematic uncertainties related to the background subtraction, a careful choice of the fit range in the event-shape distribution is needed, over which the theoretical prediction is adjusted to the data. At the other end of the spectrum (the two-jet limit) the hadronization corrections become sizeable, and correspondingly the fit range has to be adapted.
These corrections are obtained from phenomenological models implemented in Monte Carlo generators (JETSET, HERWIG, ARIADNE). The difference in the results obtained with different generators are quoted as systematic uncertainties due to hadronization, of the order of $1-2 \%$.

The dominant systematic uncertainty is related to missing higher order terms in the perturbative calculations. In order to avoid a very bad fit quality when changing the renormalization scale, and in particular to reduce the multitude and arbitrariness of prescriptions used by the LEP experiments, the LEPQCDWG has proposed a new method, the so-called uncertainty band method. First a reference prediction is chosen, such as the modified $\log \mathrm{R}$ matching scheme, together with a certain $\alpha_{s}$ value. Then various changes are applied to the prediction, keeping the same $\alpha_{s}$ value: The renormalization scale $\mu$ is varied in the range $0.5<\mu / \sqrt{s}<2$, the modified $\mathrm{R}$ matching scheme is applied, the second degree modification is tested, the logarithmic terms are changed to $L^{\prime}=\ln \left(1 /\left(x_{L} y\right)\right)$ for $2 / 3 \leq x_{L} \leq 3 / 2$, which introduces different subleading terms, and the kinematic boundary $y_{\max }$ is varied over a range given by the difference between NLO and parton shower predictions. The difference of the distributions thus obtained to the reference distribution defines an uncertainty band (Fig. 回). In the next step again the reference prediction is taken, but now $\alpha_{s}\left(M_{\mathrm{Z}}\right)$ is varied such that the changes in the prediction reproduce the spread given by the uncertainty band over the fit range. The necessary variations in $\alpha_{s}\left(M_{\mathrm{Z}}\right)$ define the theoretical uncertainty.

The LEPQCDWG has combined all measurements based on event-shape distributions at LEP1 and LEP2 for the six variables mentioned above. Correlations between variables, energies and experiments have been taken into account. The results are $\alpha_{s}\left(M_{\mathrm{Z}}\right)=0.1197 \pm$ $0.0002_{\text {stat }} \pm 0.0008_{\text {exp }} \pm 0.0010_{\text {had }} \pm_{0.0047 \text { theo }}^{0.0048}$ for LEP1 alone, $\alpha_{s}\left(M_{\mathrm{Z}}\right)=0.1196 \pm 0.0005_{\text {stat }} \pm$ $0.0010_{\exp } \pm 0.0007_{\text {had }} \pm_{0.0044 \text { theo }}^{0.0043}$ for LEP2 alone, and $\alpha_{s}\left(M_{\mathrm{Z}}\right)=0.1198 \pm 0.0003_{\text {stat }} \pm 0.0009_{\text {exp }} \pm$ $0.0008_{\text {had }} \pm 0.0046_{\text {theo }}$ for all LEP energies combined (including measurements at an effective centre-of-mass energy below $M_{\mathrm{Z}}$, obtained by 
selecting events with hard initial state radiation). Excellent consistency between the LEP1 and LEP2 measurements is found. Taking the individual measurements at the various energy points, perfect agreement with the expected running of the strong coupling is observed (Fig. 2).

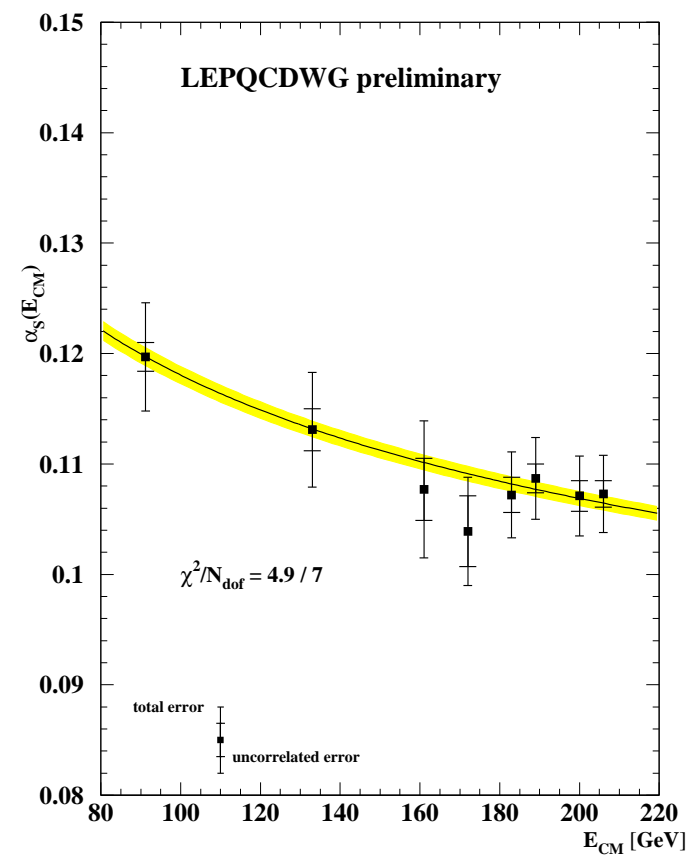

Figure 2. Combined measurements of $\alpha_{s}$ from event-shape variables at different LEP energies, compared to the expected running.

In an analysis by DELPHI the energy dependence of the mean values of event-shape variables has been analysed [5], but now the hadronization corrections are replaced by a power law as proposed in [6]. It results in a rather precise determination of $\alpha_{s}\left(M_{\mathrm{Z}}\right)=0.1184 \pm 0.0033_{\mathrm{tot}}$. A more detailed discussion of power law studies can be found in [7].

\section{Four-jet rate}

Recently, the ALEPH collaboration has published a new determination of $\alpha_{s}$ [8], based on a measurement of the four-jet rate (Durham algorithm) at LEP1. The difference to the event- shape variables is that here the perturbative expansion starts at $\mathcal{O}\left(\alpha_{s}^{2}\right)$. The full NLO corrections are known, as well as the resummation of NLL terms [9]. The quoted result is extremely precise, namely $\alpha_{s}\left(M_{\mathrm{Z}}\right)=0.1170 \pm 0.0001_{\text {stat }} \pm$ $0.0009_{\text {exp }} \pm 0.0003_{\text {had }} \pm 0.0008_{\text {theo }}$. This is mainly due to the larger sensitivity, since the leading term is of $\mathcal{O}\left(\alpha_{s}^{2}\right)$. It is observed that although the $\chi^{2}$ of the fit changes substantially when varying the renormalization scale over a wide range, and also the location of the sharp minimum in this $\chi^{2}$ varies after changes in the fitted prediction, the actually fitted $\alpha_{s}\left(M_{\mathrm{Z}}\right)$ value remains remarkably stable.

\section{Summary}

New combined results for measurements of $\alpha_{s}\left(M_{\mathrm{Z}}\right)$ at LEP have been presented, based on event-shape distributions and a new prescription for the theoretical uncertainty. Furthermore, it has turned out that the measurement of the fourjet rate at LEP allows for a very precise determination of $\alpha_{s}\left(M_{\mathrm{Z}}\right)$.

\section{Acknowledgements}

I would like to thank the members of the LEPQCDWG for providing me with the relevant numbers and plots.

\section{REFERENCES}

1. S. Bethke, J. Phys. G26 (2000) R27.

2. ALEPH Collab., Note 2002-012 CONF 2002002, ICHEP02 abs296; L3 Collab., Phys. Lett. B536 (2002) 217; DELPHI Collab., Note 2002-050-CONF-584, ICHEP02 abs229; OPAL Collab., Note PN512, ICHEP02 abs368; Note PN473, ICHEP02 abs370.

3. A. Banfi et al., JHEP 0201 (2002) 018.

4. S. Catani et al., Nucl. Phys., B407 (1993) 3.

5. DELPHI Collab., Note 2002-050-CONF-584, ICHEP02 abs229.

6. Yu.L. Dokshitzer and B.R. Webber, Phys. Lett. B352 (1995) 451.

7. P.A. Movilla Fernandez, these proceedings.

8. ALEPH Collab., CERN-EP-2002-029, accepted for publication in Eur. Phys. J. C. 
9. Z. Nagy and Z. Trócsányi, Phys. Rev. Lett. 79 (1997) 3604; Phys. Rev. D59 (1999) 014020; L. J. Dixon and A. Signer, Phys. Rev. Lett. 78 (1997) 811. 